\title{
Impact of adjuvant therapy in patients with a microscopically positive margin after resection for gastric and esophageal cancers
}

\author{
Lucy X. Ma ${ }^{1}$, Osvaldo Espin-Garcia ${ }^{2}$, Charles H. Lim ${ }^{1}$, Di M. Jiang ${ }^{1}$, Hao-Wen Sim ${ }^{1}$, Akina Natori ${ }^{1,3}$, \\ Bryan A. Chan ${ }^{1}$, Chihiro Suzuki ${ }^{1}$, Eric X. Chen ${ }^{1}$, Geoffrey Liu ${ }^{1}$, Savtaj S. Brar ${ }^{4}$, Carol J. Swallow ${ }^{4}$, \\ Jonathan C. Yeung ${ }^{5}$, Gail E. Darling ${ }^{5}$, Rebecca K. Wong ${ }^{6}$, Sangeetha N. Kalimuthu ${ }^{7}$, James Conner, \\ Elena Elimova ${ }^{1}$, Raymond W. Jang ${ }^{1}$
}

\begin{abstract}
${ }^{1}$ Department of Medical Oncology, Princess Margaret Cancer Centre, University Health Network, University of Toronto, Toronto, Canada; ${ }^{2}$ Department of Biostatistics, Princess Margaret Cancer Centre, University Health Network, University of Toronto, Toronto, Canada; ${ }^{3}$ Department of Medical Oncology, University of Miami, Miami, FL, USA; ${ }^{4}$ Department of Surgical Oncology, Princess Margaret Cancer Centre, University Health Network and Sinai Health System, University of Toronto, Toronto, Canada; ${ }^{5}$ Division of Thoracic Surgery, Department of Surgery, Toronto General Hospital, University Health Network, University of Toronto, Toronto, Canada; ${ }^{6}$ Department of Radiation Oncology, Princess Margaret Cancer Centre, University Health Network, University of Toronto, Toronto, Canada; ${ }^{7}$ Department of Pathology, Laboratory Medicine Program, University Health Network, University of Toronto, Toronto, Canada; ${ }^{8}$ Department of Pathology and Laboratory Medicine, Mount Sinai Hospital, University of Toronto, Toronto, Canada

Contributions: (I) Conception and design: LX Ma, E Elimova, RW Jang; (II) Administrative support: E Elimova, RW Jang; (III) Provision of study materials or patients: EX Chen, G Liu, SS Brar, CJ Swallow, JC Yeung, GE Darling, RK Wong, SN Kalimuthu, J Conner, E Elimova, RW Jang; (IV) Collection and assembly of data: LX Ma, CH Lim, DM Jiang, HW Sim, A Natori, BA Chan, C Suzuki; (V) Data analysis and interpretation: LX Ma, O Espin-Garcia, E Elimova, RW Jang; (VI) Manuscript writing: All authors; (VII) Final approval of manuscript: All authors.

Correspondence to: Raymond W. Jang. Department of Medical Oncology, Princess Margaret Cancer Centre, University Health Network, University of Toronto, 610 University Ave, Toronto, ON, M5G 2C1, Canada. Email: Raymond.Jang@uhn.ca.
\end{abstract}

Background: A microscopically positive (R1) resection margin following resection for gastric and esophageal cancers has been documented to be a poor prognostic factor. The optimal strategy and impact of different modalities of adjuvant treatment for an R1 resection margin remain unclear.

Methods: A retrospective analysis was performed for patients with gastric and esophageal adenocarcinoma treated at the Princess Margaret Cancer Centre (PMCC) from 2006-2016. Electronic medical records of all patients with an R1 resection margin were reviewed. Kaplan-Meier and Cox proportional hazards methods were used to analyze recurrence free survival (RFS) and overall survival (OS) with stage and neoadjuvant treatment as covariates in the multivariate analysis.

Results: We identified 69 gastric and esophageal adenocarcinoma patients with a R1 resection. Neoadjuvant chemoradiation was used in $13 \%$ of patients, neoadjuvant chemotherapy in $12 \%$, surgery alone in $75 \%$. Margins involved included proximal in $30 \%$, distal in $14 \%$, radial in $52 \%$ and multiple margins in $3 \%$ of patients. Pathological staging showed $3 \%$ with stage I disease, $20 \%$ stage II and $74 \%$ stage III. Adjuvant therapy was given in $52 \%$ of R1 pts (28\% CRT, $20 \%$ chemotherapy alone, $3 \%$ radiation alone, $1 \%$ reoperation). Median RFS was 14.1 months [95\% confidence interval (CI), 11.1-17.2]. The site of first recurrence was $72 \%$ distant, $12 \%$ mixed, 16\% locoregional alone. Median OS was 34.5 months (95\% CI, 23.3-57.9) for all patients. There was no significant difference in RFS (adjusted $\mathrm{P}=0.26$ ) or OS (adjusted $\mathrm{P}=0.83$ ) comparing modality of adjuvant therapy.

Conclusions: Most patients with positive margins after resection for gastric and esophageal cancer had advanced pathologic stage and prognosis was poor. Our study did not find improved RFS or OS with adjuvant treatment and only one patient had reresection. The main failure pattern was distant recurrence, suggesting that patients being considered for adjuvant radiotherapy (RT) should be carefully selected. Further studies are required to determine factors to select patients with good prognosis despite a positive margin, or those who may benefit from adjuvant treatment.

(C) Journal of Gastrointestinal Oncology. All rights reserved. 
Keywords: Gastric cancer; esophageal cancer; positive surgical margins

Submitted Dec 05, 2019. Accepted for publication Feb 18, 2020.

doi: 10.21037 /jgo.2020.03.03

View this article at: http://dx.doi.org/10.21037/jgo.2020.03.03

\section{Introduction}

Gastric and esophageal cancers are a major health burden globally, with high morbidity and mortality despite recent advances in therapy (1). For curative intent treatment, surgery remains an essential component of management. Contemporary therapeutic approaches often include multimodality treatment, which has been shown to improve survival and may comprise of surgery, chemotherapy and radiation.

Curative (R0) resection is defined as resection of the primary tumor without any residual microscopic or macroscopic disease, while microscopic residual tumor at the surgical margin is defined as a $\mathrm{R} 1$ resection. In both gastric and esophageal cancer, predictors of a positive margin include advanced tumor and nodal stage, tumor size, and diffuse histology (2-5). Having a positive margin has been shown to be a poor prognostic factor even after adjusting for other confounders of aggressive biology (2-10), although some studies suggest that this prognostic effect is more significant in patients with limited nodal involvement $(3-5,11,12)$.

Options for management after an R1 resection may include a combination of re-resection, adjuvant chemotherapy, radiation, or observation. Given the low incidence of a microscopically positive margin, there are no randomized trials to guide management. The optimal strategy and impact of different modalities of adjuvant treatment remain unclear, with conflicting recommendations in the literature. This study aims to examine recurrence patterns and survival outcomes in gastric and esophageal cancer patients with R1 resections and explore the impact of adjuvant treatment.

\section{Methods}

\section{Patients}

A retrospective analysis was performed for patients with gastric and esophageal adenocarcinoma treated at the Princess Margaret Cancer Centre (PMCC) from 2006-2016. Patients included had curative intent surgical resection either within the University Health Network or at an outside institution. Patients referred postoperatively from an outside institution were reviewed by a multidisciplinary team consisting of surgical oncologists, medical oncologists, radiation oncologists, radiologists and pathologists whenever possible. This study was approved by the University Health Network Research Ethics Board. Electronic medical records of all patients with an R1 resection margin were reviewed. Data was collected by trained abstractors and all extracted data was verified by a second investigator. The pathological stage was classified using the American Joint Committee on Cancer (AJCC) 7th edition TNM staging system. The presence of tumor within $1 \mathrm{~mm}$ of the surgical margin was used to define a R1 resection, as stipulated by the Royal College of Pathologists' criteria.

\section{Follow up}

Duration of follow up period was defined from the date of surgery. First recurrences were classified as local, distant or mixed based on the first site of recurrence. Local recurrence was defined as recurrence in the tumor bed, anastomotic site, or locoregional lymph node (LN) stations. Distant recurrence was defined as recurrence in other organs, peritoneum, or non-locoregional LNs. Mixed recurrence was defined as the simultaneous presence of locoregional and distant recurrence. Recurrence free survival (RFS) and overall survival (OS) were calculated as the time from the date of surgery to the date of death from any cause, or the date of detection of recurrence respectively.

\section{Statistical analysis}

The Kaplan-Meier and Cox proportional hazards methods were used to analyze RFS and OS. Due to the limited number of events, only stage and neoadjuvant treatment were included as covariates in the multivariable analysis. A subset analysis was performed to analyze esophageal (including esophageal, Siewert I and Siewert II) and gastric (including gastric and Siewert III) patients separately. One patient who had two concurrent primary tumors (Siewert II and gastric) 
was included in the analysis of the entire cohort but omitted from the subset analysis. All analyses were performed in $\mathrm{R}$ version 3.5.2 (R Core Team 2018, Vienna, Austria).

\section{Results}

\section{Patient characteristics}

Clinical and pathologic characteristics are summarized in Table 1. We identified 69 patients (16\% esophageal, $41 \%$ gastroesophageal junction, $42 \%$ gastric) with an R1 resection. Patients were predominantly male (74\%) with a median age of 65 (range, 33-86) years. Neoadjuvant chemoradiation was used in $13 \%$ of patients, neoadjuvant chemotherapy in $12 \%$, surgery alone in $75 \%$. Of the 52 patients who did not receive neoadjuvant treatment, 26 (50\%) had gastric cancer, 21 (40\%) gastroesophageal junction and $5(10 \%)$ esophageal. Reasons provided for not giving neoadjuvant included the following: 19 (37\%) were referred to the PMCC postoperatively from an outside center, 7 (13\%) were due to patient clinical factors, $3(6 \%)$ due to tumor factors such as bleeding or perforation, 3 (6\%) patient preference, 16 (31\%) unknown, 4 (8\%) other (Table 1). On pathologic staging, $83 \%$ had a pT3-4 tumor, $64 \%$ pN2-3 nodal involvement, 68\% grade 3, 70\% positive for lymphovascular invasion. Of all R1 patients, $3 \%$ were stage I, 20\% stage II, 74\% stage III. The proximal margin was involved in $30 \%$ of patients, distal $14 \%$, radial $52 \%$, and multiple margins were positive in $3 \%$.

\section{Management after $R 1$ resection}

Adjuvant therapy was given in $36(52 \%) \mathrm{R} 1$ patients (Table 2). Of these patients, 12 had received neoadjuvant treatment. Adjuvant CRT was used in $19(28 \%)$ patients, with the most common regimen being 5 -fluorouracil (5-FU) with 4,500 cGy of radiation in accordance with the MacDonald protocol (13). Chemotherapy alone was given to $14(20 \%)$ patients, with most patients receiving a triplet regimen consisting of an anthracycline, platinum and fluoropyrimidine (Table 2). Radiation alone was used in 3\% of patients. Only 1 patient had reoperation, which was also followed by CRT.

In the 33 patients who did not receive adjuvant therapy, the most common reasons were patient comorbidities/ poor performance status (21\%), complicated postoperative course prohibiting further treatment $(15 \%)$, further therapy deemed not indicated by physician (24\%), patient preference $(12 \%)$, postoperative imaging showing metastatic disease $(9 \%)$, other $(6 \%)$ and unknown (12\%) (Table 3).

\section{Recurrence and RFS}

The median follow up time was 11.3 (range, 0.6-118.3) months. A total of 50 (72\%) patients had documented recurrences. Of these, the site of first recurrence was distant in $72 \%$ of patients, mixed in $12 \%$ and locoregional alone in $16 \%$ (Figure 1). The median RFS for all patients was 14.1 months [95\% confidence interval (CI), 11.1-17.2]. There was no significant difference in RFS in patients with and without adjuvant treatment, or between modalities of adjuvant treatment groups (Figure 2 and Table 4).

\section{OS}

The median OS was 34.5 months (95\% CI, 23.3-57.9) for all patients. The 2 - and 5 -year survival rates were $61 \%(95 \%$ CI, $49-76 \%$ ) and $22 \%$ (95\% CI 10-46\%) respectively. There was no significant difference observed in the OS between patients with and without adjuvant therapy, or between different modalities of adjuvant treatment (Figure 3 and Table 5).

\section{Subset analysis of esophageal patients}

Of the esophageal cancer patients $(\mathrm{N}=33)$, the median RFS was 15.2 months (95\% CI, 5.6-20.7), with no difference in RFS with and without adjuvant therapy (adjusted $\mathrm{P}=0.11$ ) (Figure 4A). The median OS was 28.5 months (95\% CI, 22.9-57.9), with a 3 -year OS rate of $47 \%$ (95\% CI, 30 $74 \%$ ) (Figure 4B). There was no difference in OS between patients who received adjuvant treatment and those who did not (adjusted $\mathrm{P}=0.61$ ).

\section{Subset analysis of gastric patients}

Of the gastric cancer patients $(\mathrm{N}=35)$, the median RFS was 15.6 months (95\% CI, 11.5-21.8), with no difference in RFS with or without adjuvant therapy (adjusted $\mathrm{P}=0.097$ ) (Figure 5A). The median OS was 34.5 months (95\% CI, 21.4-68.7), with a 3 -year OS rate of $48 \%$ (95\% CI, 31 $75 \%$ ) (Figure $5 B$ ). There was no difference in OS between patients who received adjuvant treatment and those who did not (adjusted $\mathrm{P}=0.15$ ).

\section{Discussion}

The predisposing factors and negative prognostic effect of a microscopically positive margin after curative intent resection for gastric and esophageal cancers have been 
Table 1 Clinicopathological features of R1 patients

\begin{tabular}{|c|c|}
\hline Clinicopathological features & $\mathrm{N}=69[\%]$ \\
\hline \multicolumn{2}{|l|}{ Age (years) } \\
\hline Median [range] & $65.1[33-86]$ \\
\hline \multicolumn{2}{|l|}{ Sex } \\
\hline Male & $51[74]$ \\
\hline \multicolumn{2}{|l|}{ Tumor location ${ }^{1}$} \\
\hline Esophageal & $11[16]$ \\
\hline Siewert I & $12[17]$ \\
\hline Siewert II & $10[14]$ \\
\hline Siewert III & 6 [9] \\
\hline Gastric & 29 [42] \\
\hline Multiple & $1[1]$ \\
\hline \multicolumn{2}{|l|}{ Histology } \\
\hline Adenocarcinoma & $69[100]$ \\
\hline \multicolumn{2}{|l|}{ Neoadjuvant treatment } \\
\hline None & $52[75]$ \\
\hline Chemotherapy & 8 [12] \\
\hline Chemoradiation & $9[13]$ \\
\hline \multicolumn{2}{|c|}{ Reason for no neoadjuvant treatment $(\mathrm{N}=52)$} \\
\hline Referred to PMCC postoperatively & 19 [37] \\
\hline Patient clinical factors & $7[13]$ \\
\hline Tumor factors & $3[6]$ \\
\hline Patient preference & $3[6]$ \\
\hline Other & $4[8]$ \\
\hline Unknown & $16[31]$ \\
\hline \multicolumn{2}{|l|}{ Type of surgery } \\
\hline Esophagectomy ${ }^{2}$ & $30[43]$ \\
\hline Ivor-Lewis & 19 \\
\hline McKeown & 2 \\
\hline Left thoracoabdominal & 4 \\
\hline Transhiatal & 3 \\
\hline Pharyngolaryngoesophagectomy & 1 \\
\hline Esophagogastrectomy $^{3}$ & $5[7]$ \\
\hline Gastrectomy & 34 [49] \\
\hline Subtotal gastrectomy & 23 \\
\hline Total gastrectomy & 8 \\
\hline $\begin{array}{l}\text { Total gastrectomy with partial } \\
\text { esophagectomy }\end{array}$ & 3 \\
\hline
\end{tabular}

Table 1 (continued)

C) Journal of Gastrointestinal Oncology. All rights reserved.
Table 1 (continued)

\begin{tabular}{|c|c|}
\hline Clinicopathological features & $\mathrm{N}=69[\%]$ \\
\hline \multicolumn{2}{|l|}{ LN harvested } \\
\hline Mean \pm SD & $22.3 \pm 12.4$ \\
\hline \multicolumn{2}{|l|}{ LN positive } \\
\hline Mean \pm SD & $7.1 \pm 7.2$ \\
\hline \multicolumn{2}{|l|}{ pT classification } \\
\hline $\mathrm{T} 1$ & $1[1]$ \\
\hline $\mathrm{T} 2$ & $9[13]$ \\
\hline T3 & $42[61]$ \\
\hline T4 & $15[22]$ \\
\hline Missing & $2[3]$ \\
\hline \multicolumn{2}{|l|}{$\mathrm{pN}$ classification } \\
\hline No & $12[17]$ \\
\hline N1 & $11[16]$ \\
\hline N2 & 13 [19] \\
\hline N3 & $31[45]$ \\
\hline $\mathrm{Nx}$ & $1[1]$ \\
\hline Missing & $1[1]$ \\
\hline \multicolumn{2}{|l|}{ Pathologic stage } \\
\hline IB & $2[3]$ \\
\hline IIA & $1[1]$ \\
\hline IIB & $13[19]$ \\
\hline IIIA & $14[20]$ \\
\hline IIIB & 20 [29] \\
\hline IIIC & 17 [25] \\
\hline Missing & $2[3]$ \\
\hline \multicolumn{2}{|l|}{ Tumor grade } \\
\hline 1 & $2[3]$ \\
\hline 2 & 15 [22] \\
\hline 3 & $47[68]$ \\
\hline Not applicable $^{4}$ & $1[1]$ \\
\hline Missing & $4[6]$ \\
\hline \multicolumn{2}{|l|}{ Lymphovascular invasion } \\
\hline Present & $48[70]$ \\
\hline Absent & $14[20]$ \\
\hline Indeterminate & $3[4]$ \\
\hline Missing & $4[6]$ \\
\hline
\end{tabular}

Table 1 (continued) 
Table 1 (continued)

\begin{tabular}{lc}
\hline Clinicopathological features & $\mathrm{N}=69[\%]$ \\
\hline Margin involved & \\
Proximal & $21[30]$ \\
Distal & $10[14]$ \\
Radial & $36[52]$ \\
Multiple & $2[3]$ \\
\hline
\end{tabular}

${ }^{1}$, Siewert class based on epicenter of tumor, with the anatomical cardia defined as $40 \mathrm{~cm} .<35 \mathrm{~cm}=$ esophageal, $35-39 \mathrm{~cm}=$ Siewert I, 39-42 cm = Siewert II, 42-45 cm = Siewert III, $>45 \mathrm{~cm}$ $=$ gastric ${ }^{2}$, one patient documented in clinical notes to have esophagectomy, unknown approach; ${ }^{3}$, five patients who had surgery at an outside institution with "esophagogastrectomy" documented in clinical notes and pathology report, no operative report available; ${ }^{4}$, post-treatment changes. PMCC, Princess Margaret Cancer Centre; LN, lymph node.

Table 2 Treatment after $\mathrm{R} 1$ resection

\begin{tabular}{lc}
\hline Treatment & $\mathrm{N}=69[\%]$ \\
\hline Reoperation and CRT & $1[1]$ \\
Chemotherapy & $14[20]$ \\
ECF/ECX/EOX & 9 \\
Cisplatin/5FU & 2 \\
Other & 3 \\
CRT & $19[28]$ \\
$5 F U$ & 13 \\
Cisplatin/5FU & 3 \\
Carboplatin/paclitaxel & 2 \\
Other & 1 \\
Radiation & $2[3]$ \\
No adjuvant & $33[48]$
\end{tabular}

CRT, chemoradiation; ECF, epirubicin, cisplatin and 5-fluorouracil; ECX, epirubicin, cisplatin and capecitabine; EOX, epirubicin, oxaliplatin and capecitabine; 5-FU, 5-fluorouracil.

shown in many studies. Our data support that having a positive margin is likely a marker of underlying aggressive tumor biology, as reflected by the advanced pathologic features of the patients in our cohort.

The ideal management of positive margins remains controversial, with no prospective randomized trials to direct therapeutic decisions. Patients with positive
Table 3 Reason for no adjuvant treatment

\begin{tabular}{lc}
\hline Reason & $\mathrm{N}=33[\%]$ \\
\hline Patient clinical status & $7[21]$ \\
Postoperative complications prohibiting treatment & $5[15]$ \\
Deemed not indicated by oncologist & $8[24]$ \\
Patient preference & $4[12]$ \\
Metastatic disease on postoperative imaging & $3[9]$ \\
Other & $2[6]$ \\
Unknown & $4[12]$ \\
\hline
\end{tabular}

margins were often excluded from landmark trials that guide contemporary management such as the Intergroup 0116, ARTIST and ARTIST 2 studies which investigated the role of postoperative chemoradiotherapy, as well as the CLASSIC trial which examined the use of adjuvant chemotherapy in patients with gastric cancer (13-16).

The current National Comprehensive Cancer Network (NCCN) guidelines place an emphasis on postoperative locoregional therapy modalities for $\mathrm{R} 1$ patients, recommending chemoradiation for patients who did not receive it preoperatively and consideration of re-resection if feasible in patients who already received neoadjuvant therapy. The writers of the guideline acknowledge that this strategy has not been evaluated in prospective trials, but felt that this was a "reasonable treatment option given the significantly worse prognosis associated with margin-positive resections, especially in patients who have not received preoperative therapy" $(17,18)$. There is no recommendation for or against postoperative chemotherapy alone for R1 patients.

The majority of the literature on postoperative management of $\mathrm{R} 1$ patients is retrospective, with conflicting reports and recommendations regarding the role of different modalities of adjuvant therapy. Some studies align with the NCCN guideline and advocate for the use of postoperative chemoradiation. In a subgroup analysis of a retrospective cohort study that included $22 \mathrm{R} 1$ patients, adjuvant chemoradiation was associated with improved OS compared with surgery alone (19). Stiekema et al. found that in a cohort of 110 gastric cancer patients, 30 of whom had an R1 resection, there was no difference in survival outcomes between R1 and R0 patients after postoperative chemoradiation (20).

Conversely, others have suggested that there is no role for adjuvant radiation. In a cohort of 68 esophageal R1 patients, Vadhwana et al. did not find any RFS or OS benefit 

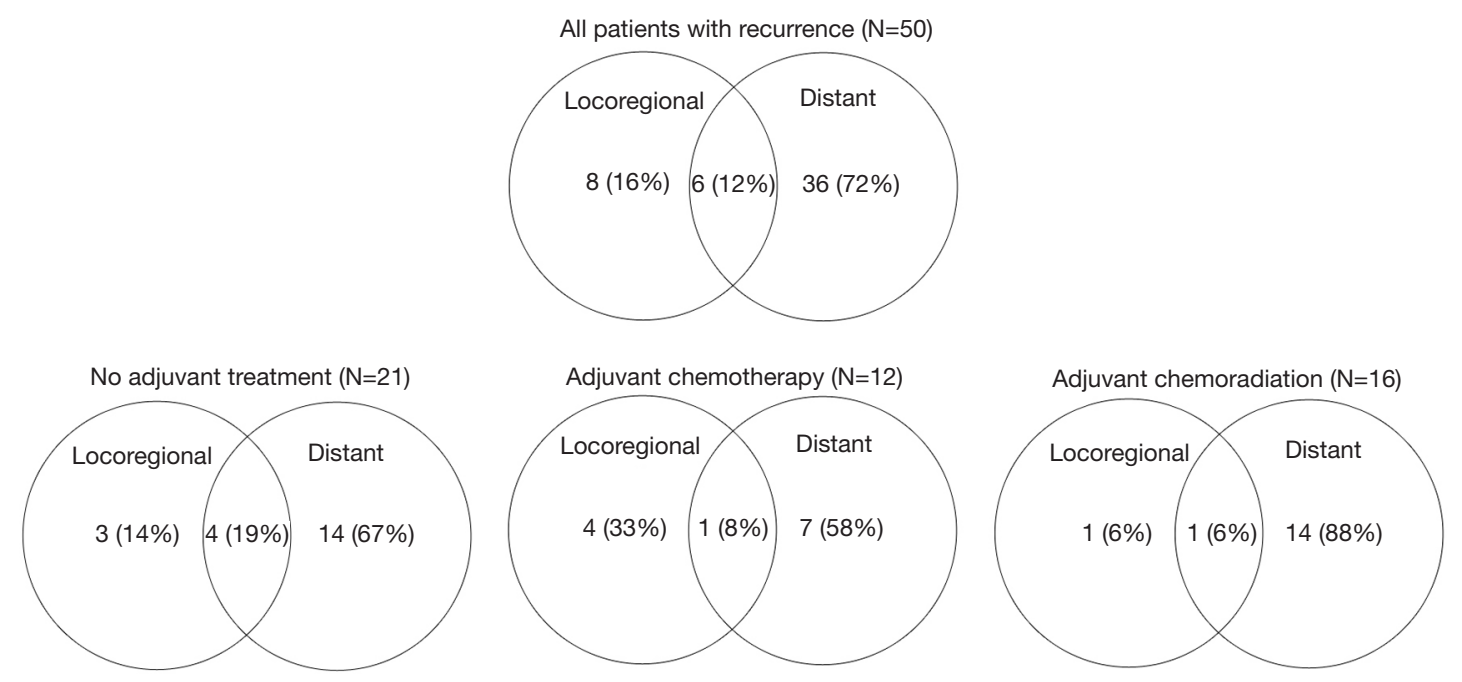

Figure 1 Location of first recurrence in R1 resection patients.
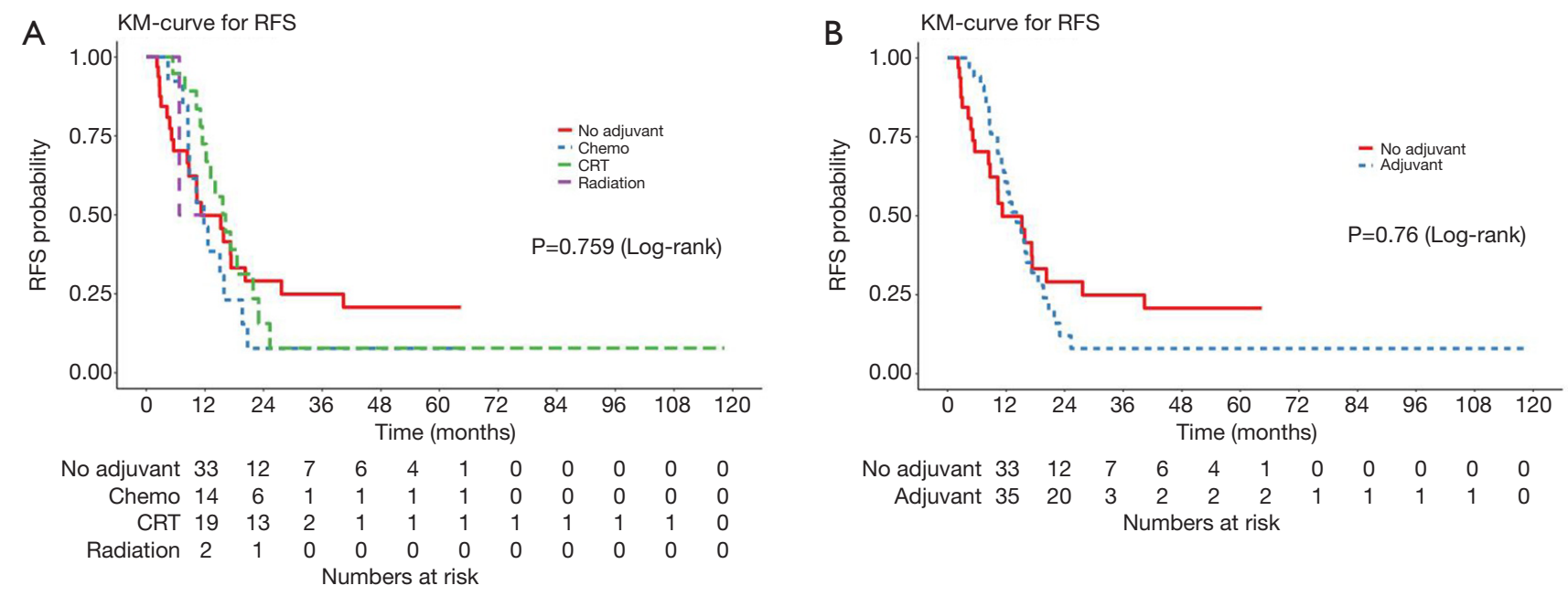

Figure 2 RFS for R1 patients (A) RFS for R1 patients by adjuvant treatment modality; (B) RFS of R1 patients with and without adjuvant treatment. RFS, recurrence free survival.

in patients who received postoperative radiation compared to those who did not (21).

Others have compared postoperative chemoradiation with chemotherapy alone. In a large study using the National Cancer Database, Rhome et al. found that concurrent chemoradiation was associated with a higher OS compared to chemotherapy alone in gastric R1 patients (22). Similarly, Zhou et al. found a trend toward benefit of chemoradiation compared to chemotherapy in a cohort of 114 patients, although this did not reach statistical significance (23). In both of these studies, there was no direct comparison to outcomes to patients who did not receive any adjuvant therapy. Contrary to these studies, Markar et al. found that postoperative chemotherapy improved OS compared to no treatment, but the addition of radiation did not provide further survival benefit (10).

Finally, others such as Gertler et al. did not find a survival benefit for $\mathrm{R} 1$ patients who underwent additive postoperative therapy (24). Our study also did not find significant improvement in RFS or OS with adjuvant 
Table 4 Univariable and multivariable analyses for RFS

\begin{tabular}{|c|c|c|c|c|c|c|}
\hline Adjuvant treatment modality & \multicolumn{3}{|c|}{ Univariable } & \multicolumn{3}{|c|}{ Multivariable } \\
\hline No adjuvant treatment & Reference & & 0.76 & Reference & & 0.26 \\
\hline Chemotherapy & $1.37(0.67-2.82)$ & 0.39 & & $0.80(0.37-1.75)$ & 0.58 & \\
\hline Chemoradiation & $0.93(0.48-1.83)$ & 0.84 & & $0.46(0.22-1)$ & 0.049 & \\
\hline
\end{tabular}

RFS, recurrence free survival; $\mathrm{HR}$, hazard ratio; $\mathrm{Cl}$, confidence interval.
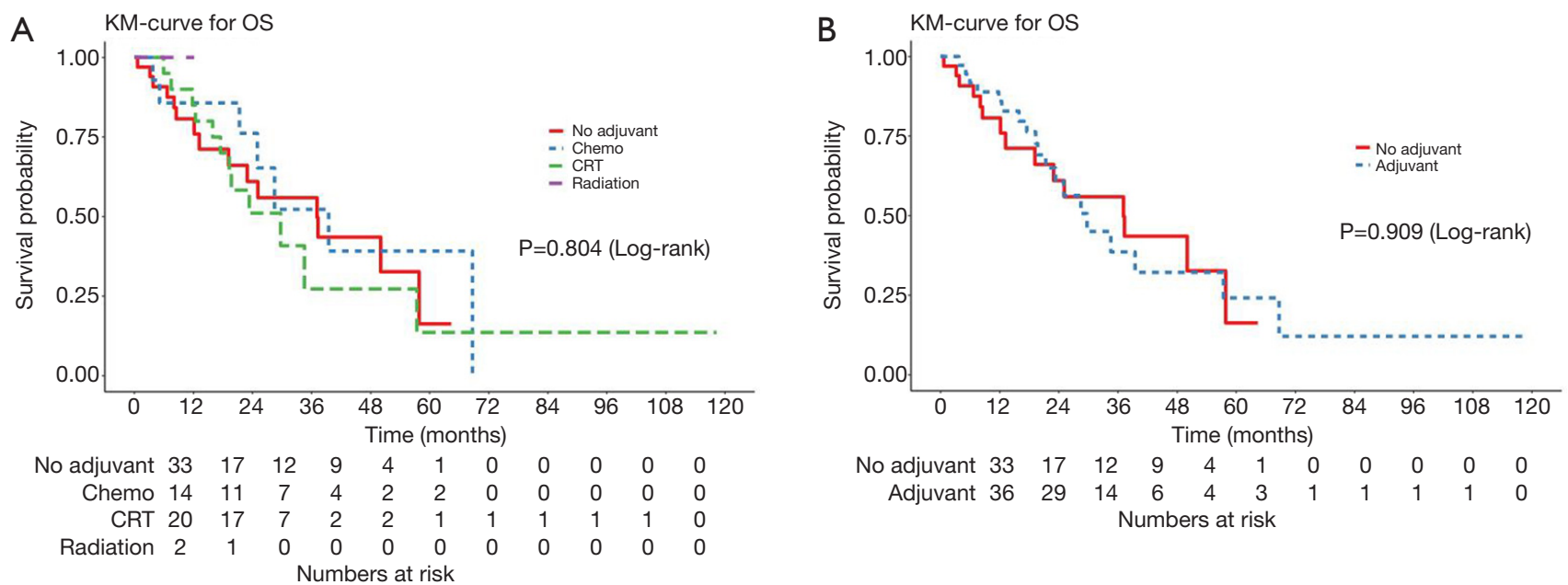

Figure 3 OS for R1 patients. (A) OS for R1 patients by adjuvant treatment modality; (B) OS of R1 patients with and without adjuvant treatment. OS, overall survival.

Table 5 Univariable and multivariable analyses for OS

\begin{tabular}{|c|c|c|c|c|c|c|}
\hline Adjuvant treatment modality & \multicolumn{3}{|c|}{ Univariable } & \multicolumn{3}{|c|}{ Multivariable } \\
\hline No adjuvant treatment & Reference & & 0.89 & Reference & & 0.83 \\
\hline Chemotherapy & $0.79(0.32-1.95)$ & 0.6 & & $0.68(0.26-1.73)$ & 0.42 & \\
\hline Chemoradiation & $1.15(0.53-2.49)$ & 0.73 & & $1.05(0.47-2.39)$ & 0.9 & \\
\hline
\end{tabular}

OS, overall survival; $\mathrm{HR}$, hazard ratio; $\mathrm{Cl}$, confidence interval.

treatment in patients with an $\mathrm{R} 1$ resection, regardless of modality of therapy.

These varying findings and recommendations from the literature emphasize the need for further studies into the behavior of R1 patients to better risk stratify and select for patients who may benefit from adjuvant treatment. Those who advocate for postoperative chemoradiation after an $\mathrm{R} 1$ resection intuitively aim to prevent locoregional disease recurrence; however, in our patient population, the main failure pattern was distant recurrence. At the time of first diagnosis of disease recurrence, $84 \%$ of our patients had distant or mixed recurrence while only $16 \%$ had an isolated 

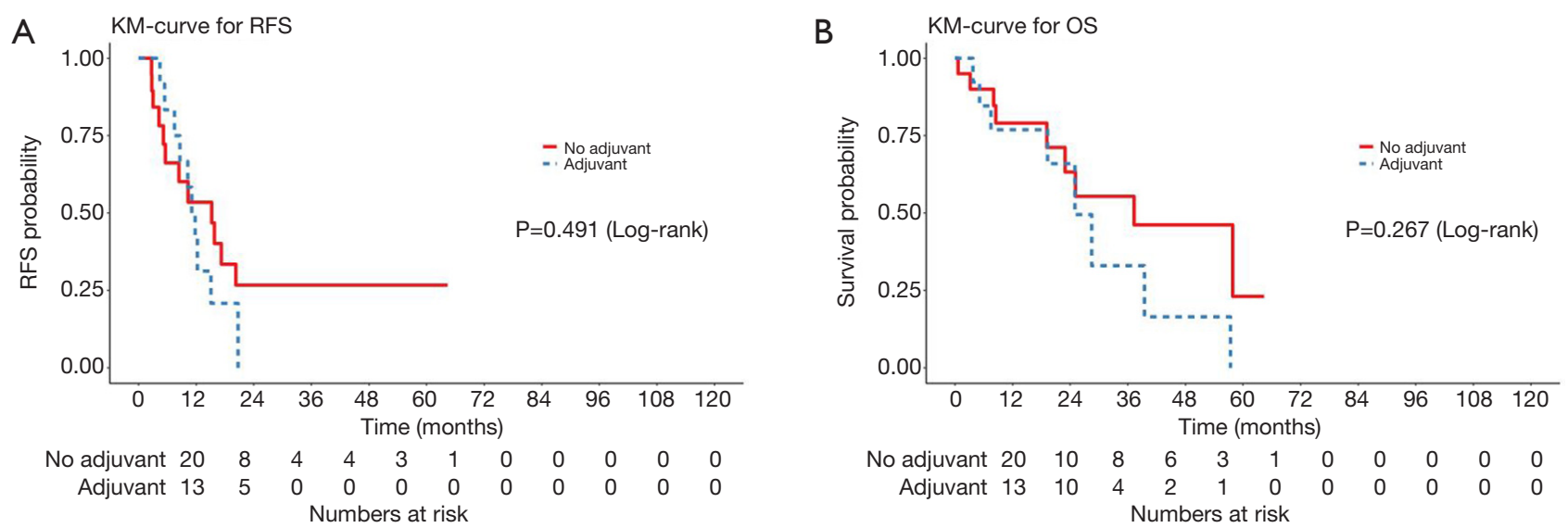

Figure 4 Survival analysis for esophageal R1 patient subset. (A) RFS for esophageal R1 patients with and without adjuvant treatment; (B) OS for esophageal R1 patients with and without adjuvant treatment. RFS, recurrence free survival; OS, overall survival.
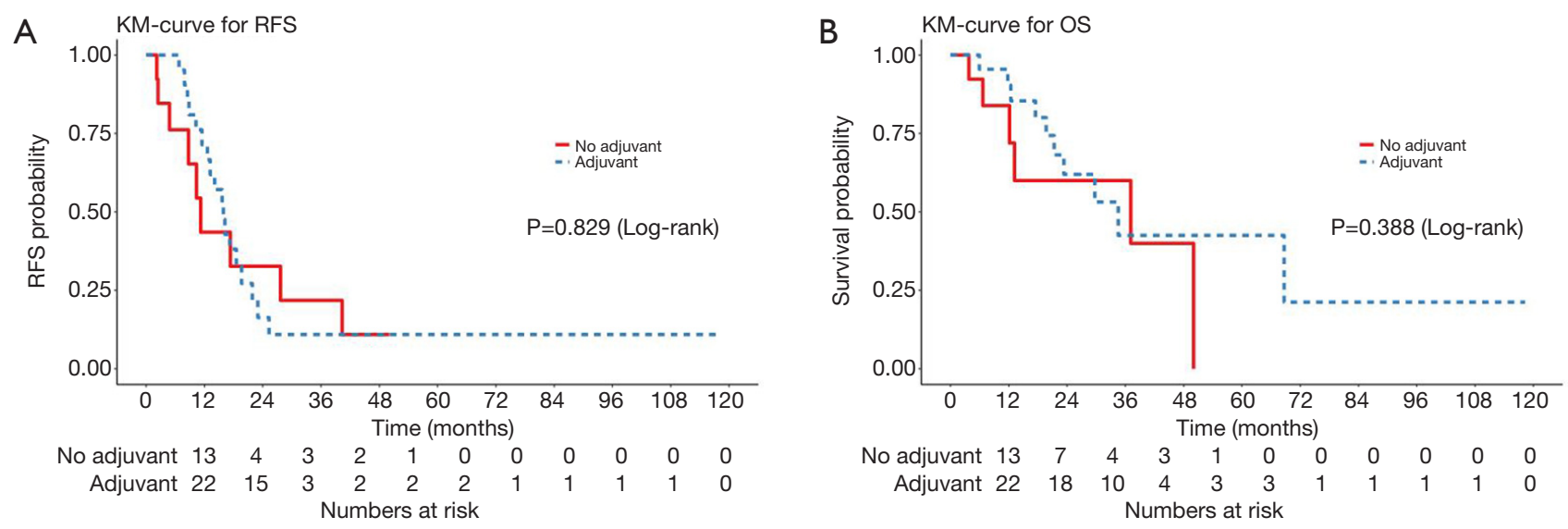

Figure 5 Survival analysis for gastric R1 patient subset. (A) RFS for gastric R1 patients with and without adjuvant treatment; (B) OS for gastric R1 patients with and without adjuvant treatment. RFS, recurrence free survival; OS, overall survival.

locoregional recurrence. Gertler et al. found a similar pattern, with $88 \%$ of patients in their cohort experiencing distant metastatic disease at the first-time disease recurrence. These recurrence patterns suggest that $\mathrm{R} 1$ resections occur in patients with aggressive biology, some of whom may even have undetected metastatic disease at the time of surgery. In these cases, postoperative locoregional therapy with radiation would not be expected to provide a survival benefit. As such, patients being considered for adjuvant radiotherapy (RT) should be carefully selected, given the high rate of systemic relapse.

Despite the poor prognosis associated with R1 status, our patient cohort did have a few patients who were long term survivors. More studies are needed to better characterize clinicopathologic factors associated with different patterns of recurrence in order to identify patients with good prognosis despite a positive margin, and to aid in decisionmaking around adjuvant treatment.

We acknowledge the limitations of our study including the retrospective and observational design and selection bias of the included patients. One surprising finding was that the majority of patients did not receive neoadjuvant treatment. Of these, half had gastric cancer and it is possible that in some of these cases, the surgeon performed upfront surgery with the intention of referring for adjuvant chemoradiation with the MacDonald protocol. Other limitations include the variation in adjuvant chemotherapy and radiation regimens, the limited sample size in a single-center cohort, differences 
in stage, and other unadjusted confounders which prevents us from making definitive recommendations. Notwithstanding these limitations, our data highlight an important knowledge gap, a significant predilection for distant rather than local recurrence in R1 disease, and the need for prospective studies to clarify the optimal adjuvant strategy.

\section{Conclusions}

Most patients with positive margins after resection for gastric and esophageal cancers had advanced pathologic features and poor prognosis. We did not find benefit in RFS or OS with adjuvant treatment. Most patients with recurrent disease had distant recurrences, suggesting that patients being considered for adjuvant locoregional therapy should be carefully evaluated. Further studies are required to determine factors to select patients with good prognosis despite R1 resection, or those who may benefit from adjuvant treatment.

\section{Acknowledgments}

Funding: None.

\section{Footnote}

Conflicts of Interest: All authors have completed the ICMJE uniform disclosure form (available at http://dx.doi. org/10.21037/jgo.2020.03.03). The authors have no conflicts of interest to declare.

Ethical Statement: The authors are accountable for all aspects of the work in ensuring that questions related to the accuracy or integrity of any part of the work are appropriately investigated and resolved. This study was approved by the Research Ethics Board of the University Health Network (approval number: 14-8075.9).

Open Access Statement: This is an Open Access article distributed in accordance with the Creative Commons Attribution-NonCommercial-NoDerivs 4.0 International License (CC BY-NC-ND 4.0), which permits the noncommercial replication and distribution of the article with the strict proviso that no changes or edits are made and the original work is properly cited (including links to both the formal publication through the relevant DOI and the license). See: https://creativecommons.org/licenses/by-nc- $\mathrm{nd} / 4.0 /$.

\section{References}

1. Ferlay J, Colombet M, Soerjomataram I, et al. Estimating the global cancer incidence and mortality in 2018: GLOBOCAN sources and methods. Int J Cancer 2019;144:1941-53.

2. Morgagni P, Garcea D, Marrelli D, et al. Resection line involvement after gastric cancer surgery: clinical outcome in nonsurgically retreated patients. World J Surg 2008;32:2661-7.

3. Sun Z, Li DM, Wang ZN, et al. Prognostic significance of microscopic positive margins for gastric cancer patients with potentially curative resection. Ann Surg Oncol 2009;16:3028-37.

4. Raziee HR, Cardoso R, Seevaratnam R, et al. Systematic review of the predictors of positive margins in gastric cancer surgery and the effect on survival. Gastric Cancer 2012;15 Suppl 1:S116-24.

5. Griffiths EA, Brummell Z, Gorthi G, et al. The prognostic value of circumferential resection margin involvement in oesophageal malignancy. Eur J Surg Oncol 2006;32:413-9.

6. Woo JW, Ryu KW, Park JY, et al. Prognostic impact of microscopic tumor involved resection margin in advanced gastric cancer patients after gastric resection. World J Surg 2014;38:439-46.

7. Chao YK, Yeh CJ, Chang HK, et al. Impact of circumferential resection margin distance on locoregional recurrence and survival after chemoradiotherapy in esophageal squamous cell carcinoma. Ann Surg Oncol 2011;18:529-34.

8. Wu J, Chen QX, Teng LS, et al. Prognostic significance of positive circumferential resection margin in esophageal cancer: a systematic review and meta-analysis. Ann Thorac Surg 2014;97:446-53.

9. Gilbert S, Martel AB, Seely AJ, et al. Prognostic significance of a positive radial margin after esophageal cancer resection. J Thorac Cardiovasc Surg 2015;149:54855; discussion 555 .

10. Markar SR, Gronnier C, Duhamel A, et al. Significance of microscopically incomplete resection margin after esophagectomy for esophageal cancer. Ann Surg 2016;263:712-8.

11. Kim SH, Karpeh MS, Klimstra DS, et al. Effect of microscopic resection line disease on gastric cancer survival. J Gastrointest Surg 1999;3:24-33.

12. Bickenbach KA, Gonen M, Strong V, et al. Association of positive transection margins with gastric cancer survival 
and local recurrence. Ann Surg Oncol 2013;20:2663-8.

13. Macdonald JS, Smalley SR, Benedetti J, et al.

Chemoradiotherapy after surgery compared with surgery alone for adenocarcinoma of the stomach or gastroesophageal junction. N Engl J Med 2001;345:725-30.

14. Lee J, Lim DH, Kim S, et al. Phase III trial comparing capecitabine plus cisplatin versus capecitabine plus cisplatin with concurrent capecitabine radiotherapy in completely resected gastric cancer with D2 lymph node dissection: the ARTIST trial. J Clin Oncol 2012;30:268-73.

15. Park SH, Zang DY, Han B, et al. ARTIST 2: Interim results of a phase III trial involving adjuvant chemotherapy and/or chemoradiotherapy after D2-gastrectomy in stage II/III gastric cancer (GC). 2019;37:4001.

16. Bang YJ, Kim YW, Yang HK, et al. Adjuvant capecitabine and oxaliplatin for gastric cancer after D2 gastrectomy (CLASSIC): a phase 3 open-label, randomised controlled trial. Lancet 2012;379:315-21.

17. National Comprehensive Cancer Network. Gastric cancer (version 2.2019). Available online: https://www.nccn.org/ professionals/physician_gls/pdf/gastric.pdf. Accessed July 30, 2019.

18. National Comprehensive Cancer Network. Esophageal and esophagogastric junction cancers (version 2.2019). Available online: https://www.nccn.org/professionals/

Cite this article as: Ma LX, Espin-Garcia O, Lim CH, Jiang DM, Sim HW, Natori A, Chan BA, Suzuki C, Chen EX, Liu G, Brar SS, Swallow CJ, Yeung JC, Darling GE, Wong RK, Kalimuthu SN, Conner J, Elimova E, Jang RW. Impact of adjuvant therapy in patients with a microscopically positive margin after resection for gastric and esophageal cancers. J Gastrointest Oncol 2020;11(2):356-365. doi: 10.21037/ jgo.2020.03.03 physician_gls/pdf/esophageal.pdf. Accessed July 30, 2019.

19. Dikken JL, Jansen EP, Cats A, et al. Impact of the extent of surgery and postoperative chemoradiotherapy on recurrence patterns in gastric cancer. J Clin Oncol 2010;28:2430-6.

20. Stiekema J, Trip AK, Jansen EP, et al. The prognostic significance of an $\mathrm{R} 1$ resection in gastric cancer patients treated with adjuvant chemoradiotherapy. Ann Surg Oncol 2014;21:1107-14.

21. Vadhwana B, Zosimas D, Lykoudis PM, et al. Adjuvant radiotherapy in oesophageal cancer with positive circumferential resection margins-recurrence and survival outcomes. J Gastrointest Oncol 2019;10:483-91.

22. Rhome RM, Moshier E, Sarpel U, et al. Predictors of positive margins after definitive resection for gastric adenocarcinoma and impact of adjuvant therapies. Int J Radiat Oncol Biol Phys 2017;98:1106-15.

23. Zhou ML, Li GC, Yang W, et al. Adjuvant chemoradiotherapy versus adjuvant chemotherapy for R1 resected gastric cancer: a retrospective cohort study. Br J Radiol 2018;91:20180276.

24. Gertler R, Richter J, Stecher L, et al. What to do after R1-resection of adenocarcinomas of the esophagogastric junction? J Surg Oncol 2016;114:428-33. 